\title{
Tumor Vascular Invasion
}

National Cancer Institute

\section{Source}

National Cancer Institute. Tumor Vascular Invasion. NCI Thesaurus. Code C35939.

A term referring to the presence of tumor emboli or tumor masses within any circulatory vessel. 